\title{
Free-Volume Evolution of 1-Propanol Confined in Variously Filled Regular Mesopores of SBA-15 Matrix
}

\author{
O. ŠAuŠA ${ }^{a, *}$, M. LukeŠOVÁ ${ }^{b}$, H. ŠVAJDLEnKOVÁ ${ }^{b}$ AND J. BARTOŠ ${ }^{b}$ \\ ${ }^{a}$ Institute of Physics, Slovak Academy of Sciences, Dúbravská cesta 9, 84511 Bratislava, Slovakia \\ ${ }^{b}$ Polymer Institute, Slovak Academy of Sciences, Dúbravská cesta 9, 84541 Bratislava, Slovakia
}

\begin{abstract}
The free-volume of 1-propanol (1-PrOH) confined in a regular mesoporous SBA-15 matrix was investigated by desorption experiment at room temperature and over a wide temperature range, from $15 \mathrm{~K}$ up to $350 \mathrm{~K}$, for four different contents of 1-PrOH in the pores. The gradual desorption of 1-PrOH from the mesopores as well as the free volume temperature dependences have shown that the polar molecules of 1-PrOH located at the inner polar surface of the SBA-15 matrix are more strongly bound to the matrix surface. The subsequent discussion of the annihilation and free-volume characteristics follows.
\end{abstract}

DOI: 10.12693/APhysPolA.132.1572

PACS/topics: positron annihilation, 1-propanol, confined systems, free-volume

\section{Introduction}

1-propanol is a polar substance with a simple structure and significant use in synthetic, solution organic and polymer chemistry. It serves as an important prototypical polar protic organic compound in various types of condensed matter studies.

The aim of this work is to show the changes in the annihilation behavior of ortho-positronium (o-Ps) in 1$\mathrm{PrOH}$ confined in regular mesopores of the SBA-15 matrix compared to the bulk state [1] as a function of different amounts of 1-PrOH in the pores, as well as of the temperature. As is known, the SBA-15 matrix is a hard inorganic matrix with a polar inner surface [2]. Therefore, we expect the effect of the attractive interactions between the polar groups of 1-propanol and the SBA15 matrix, as well as in the free-volume microstructure of the confined 1-propanol. Similar work was done with confined media by the partial filling of pores with water $[3,4]$ or $n$-hexadecane $[5,6]$ in irregular matrices. However, these substances crystallize in the bulk state, unlike the amorphous 1-PrOH, which is studied in this work.

\section{Material and equipment}

The matrix 1D-Hexagonal SBA-15 (MK Impex Corp., Canada) was used. The main characteristics of the matrix are an average pore size of $r=8.5 \mathrm{~nm}$, a surface area of SA $=718 \mathrm{~m}^{2} / \mathrm{g}$ and a total pore volume of $V_{\text {tot }}=0.93 \mathrm{~cm}^{3} / \mathrm{g}$. These quantities were determined according to the Brunauer-Emmett-Teller (BET) theory.

1-propanol (1-PrOH) from Sigma-Aldrich, Inc, Germany, of $99 \%$ purity was used as a medium. The glass

\footnotetext{
*corresponding author; e-mail: ondrej.sausa@savba.sk
}

transition temperature is $T_{q}^{D S C}=100 \mathrm{~K}$ [7]. Other characteristics of the bulk 1-PrOH can be found in work [1].

The content of 1-PrOH in the pores was determined by weighing $( \pm 0.1 \%)$ and expressed by the coefficient of filling $k$ defined as $k=m_{\text {alc }} /\left(m_{\text {matrix }}+m_{\text {alc }}\right)$, where $m_{\text {alc }}$ is the weight of $1-\mathrm{PrOH}$ and $m_{\text {matrix }}$ is the weight of the pure SBA-15. The fractional filling $f$ of the total pore volume in $\%$ is another useful quantity and can be expressed by the coefficient $k$ as $f=100 k /\left((1-k) V_{\text {tot }} \rho\right)$ where $V_{\text {tot }}$ is the total pore volume per gram of matrix and $\rho$ is the density of $1-\mathrm{PrOH}$.

The fast-fast lifetime spectrometer with the resolution about 350 ps (FWHM) was used for the annihilation experiments. The standard sandwiched geometry of the sample-source assembly was applied. The resolution function was determined by $\mathrm{Al}$ defect-free sample. The correction to annihilation in the Kapton foil was taken into account. LT program [8] was used for the analysis of spectra. Four lifetime components were fitted during this analysis. Large lifetimes $\tau_{3}$ or $\tau_{4}$ (in nanosecond range) originated from the annihilation of ortho-positronium (oPs) in free volumes of different sizes are important for this study and will be discussed later. From the $o$-Ps lifetimes, the free-volume pore sizes were estimated using EELViS routine [9] for the spherical- or cylindrical-shaped cavity approximation.

Temperature measurements in the range of 15 $350 \mathrm{~K}$ were made by the closed cycle He-refrigerator JANIS CCS-450 System with temperature stability about $\pm 0.2 \mathrm{~K}$.

\section{Results and discussion}

In the first step, desorption of 1-PrOH from SBA-15 matrix at room temperature (298 K) was studied where we focused on the microscopic free-volume, $v_{h}$, obtained from $o$-Ps lifetime measurements. The results of desorption experiment are in Figs. 1 and 2. Figure 1 displays the $o$-Ps lifetime dependence of small cavities (associated 
with 1-PrOH, $\tau_{3}$ ) and large holes (predominantly pores in the SBA matrix, $\tau_{4}$ ) as a function of the $1-\mathrm{PrOH}$ content in the SBA-15 expressed by coefficient $k$. In Fig. 2, the dependences of the relative intensities $I_{3}$ and $I_{4}$ as a function of filling $k$ show the relative representation of the large and small free volumes. From both figures we can deduce the following facts.

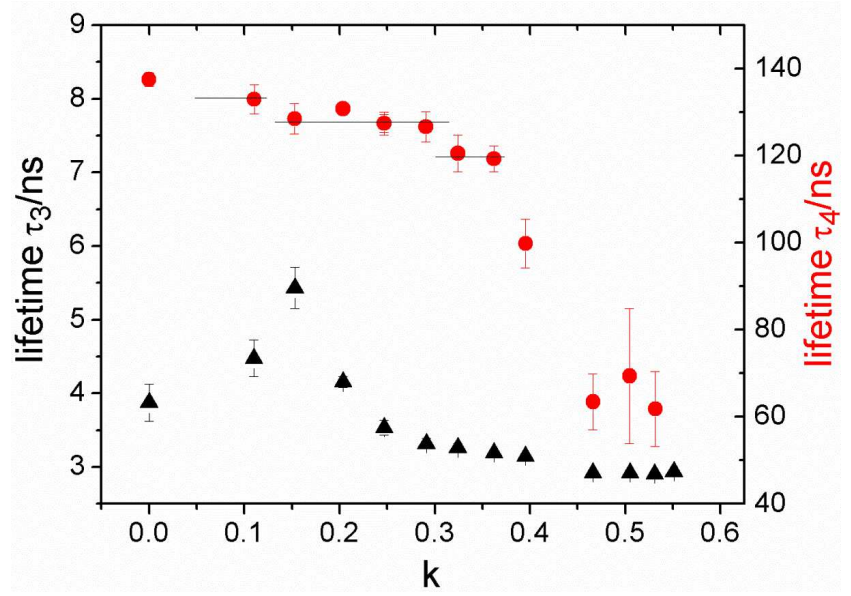

Fig. 1. Desorption of 1-propanol from SBA-15. o-Ps lifetimes $\tau_{3}$ (triangles) and $\tau_{4}$ (circles) as a function of $k$.

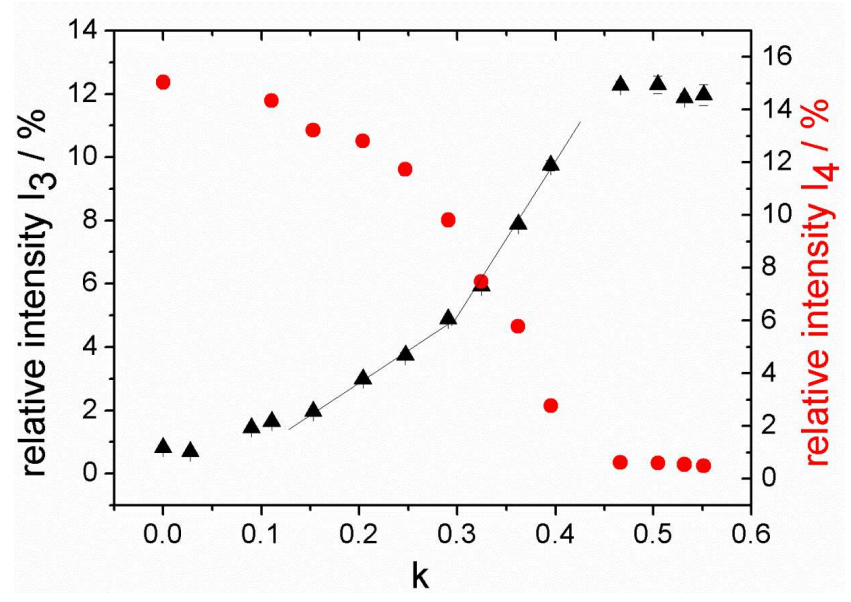

Fig. 2. Desorption of 1-propanol from SBA-15. Relative intensities $I_{3}$ (triangles) and $I_{4}$ (circles) as a function of $k$.

From the over-filled sample (part 1-PrOH was out of the fully-filled pore), a defined amount of 1-PrOH was gradually evaporated and then the sample container was hermetically closed. For $k \geq 0.42$ the annihilation is predominantly in small free volumes which are typical for liquid 1-PrOH. The annihilation in large holes is negligible (minimal $I_{4}$ ), perhaps, comes from the space between grains of SBA-15. The mesopores of matrix are full-filled. At the desorption of 1-PrOH from mesopores for $k$ in the range from 0.42 up to 0.38 , the large pores were ap- peared and started to grow fast. The compact mass of 1-PrOH in the mesopores probably disrupts quickly by forming a cylindrical free-volume in the middle of the pore. Upon further desorption of 1-PrOH for $k<0.35$, the thickness of the layer of $1-\mathrm{PrOH}$ on the internal pore wall decreased. There is a hint of a stairway structure of $\tau_{4}(k)$ dependence that shows the quasi-stepwise character which could be associated with the two- and the monolayer structure of 1-PrOH.

The size of smaller pores is associated with $\tau_{3}$. This lifetime increases slowly as the filling coefficient decreases from $k=0.42$ to 0.25 . This increase is probably connected with the creation of small cracks in the medium. Below $k=0.25$, strong tearing of the compact medium layer probably started with generating larger free-volumes than in compact 1-PrOH at high fillings. The gaps are created inside of the medium and perhaps to a lesser extent between the matrix internal surface and molecules of the 1-PrOH. The influence of opening micropores between the main cylindrical pores of the matrix at very low 1-PrOH fillings can be also investigated.

Based on these desorption findings, a series of coefficients $k$ were selected that could be related to the various 1-PrOH arrangements in the SBA-15 mesopores. These fillings were used to measure the temperature dependence of lifetimes.

In Fig. 3, the temperature dependences of the $o$-Ps lifetime $\tau_{3}$ as well as $v_{h}$ of the confined 1-PrOH for different $k$ are displayed. The dependence for the bulk $1-\mathrm{PrOH}$ is also shown for the comparison. Important temperatures are indicated by arrows.

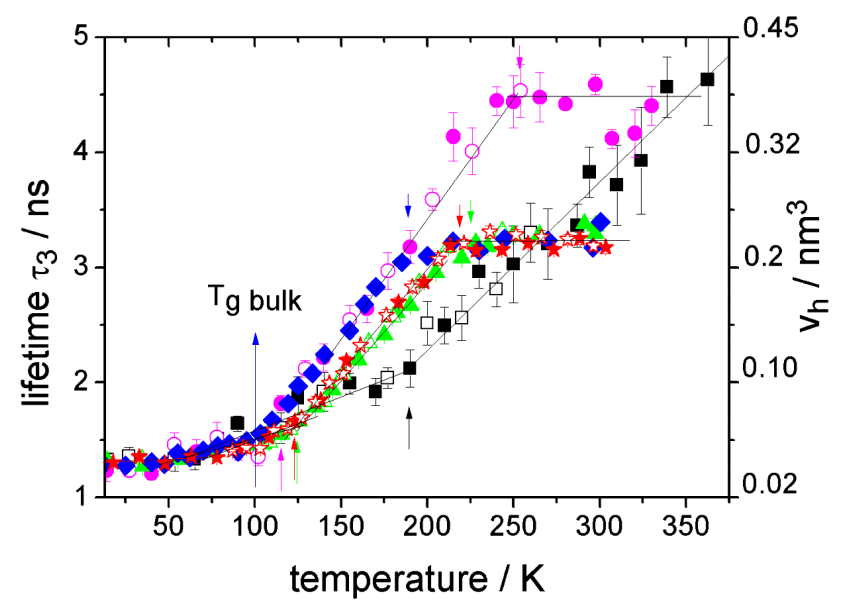

Fig. 3. Lifetime $\tau_{3}$ temperature dependence for 1propanol confined in SBA-15 matrix for the different filling coefficient $k$ (0.45 stars, 0.34 triangles, 0.25 circles, 0.15 squares) and bulk sample (diamonds). Full symbols - heating, open symbols - cooling. Arrow up - glass transition temperature $T_{g}$, arrow down onset temperature of plateau $T_{b 2}$.

The basic values of the measured free-volume characteristics are in Table I, the values for the bulk 1-PrOH were taken from work [1]. From the results it follows that 


\section{TABLE I}

The glass transition temperature $T_{g}$, onset temperature of the plateau $T_{b 2}$, free-volume at $T_{g}$ and expansion coefficient $\alpha_{s c l}$ of free-volume at $T_{g}$ for the supercooled liquid state for a series of the samples with different content of 1-propanol.

\begin{tabular}{c|c|c|c|c}
\hline \hline $\begin{array}{c}\text { Sample } \\
\text { with } k\end{array}$ & $\begin{array}{c}T_{g} \\
{[\mathrm{~K}]}\end{array}$ & $\begin{array}{c}T_{b 2} \\
{[\mathrm{~K}]}\end{array}$ & $\begin{array}{c}v_{h}\left(T_{g}\right) \\
{\left[\mathrm{nm}^{3}\right]}\end{array}$ & $\begin{array}{c}\alpha_{s c l} \\
{\left[\times 10^{-4} \mathrm{~K}^{-1}\right]}\end{array}$ \\
\hline bulk [1] & 101 & 180 & 0.052 & 334 \\
\hline 0.45 & 121 & 219 & 0.062 & 268 \\
\hline 0.34 & 121 & 224 & 0.055 & 302 \\
\hline 0.25 & 116 & 253 & 0.067 & 349 \\
\hline 0.15 & 181 & - & 0.103 & 151
\end{tabular}

large fillings (fully-filled pores at $k=0.45$ and highly filled pores at $k=0.34$ ) move the glass transition temperature $T_{g}$ to higher values. The confined 1-PrOH needs more energy for the transition from the glassy state to the supercooled liquid state. Similarly, the onset temperature $T_{b 2}$ to the plateau region, where the $o$-Ps lifetime is a quasi-constant [1], is shifted to a higher temperature compared to bulk state. In this plateau region confined $1-\mathrm{PrOH}$ for all these pore fillings as well as $1-\mathrm{PrOH}$ in bulk state have the comparable lifetimes.

On the other hand, at low degrees of fillings, the temperature dependences are dramatically different. For $k=0.25$, there are conditions to create additional freevolumes between 1-PrOH molecules that expand similarly as free-volumes in bulk or at high filling. The average value of these additional free volumes can be estimated for example at onset $T_{b 2}$ temperature from the dependence $v_{h}(T)$ for fully-filled pores $(k=0.45)$ and $v_{h}$ $(219 \mathrm{~K})$ for $k=0.25$. The difference of these free volumes is about $0.09 \mathrm{~nm}^{3}$.

For the coefficient $k=0.15$, the temperature $T_{g}$ moved to higher values where the plateau disappeared. This is due to the strong H-binding between the thin layer of polar 1-PrOH and the polar surface of the SBA-15 matrix. It requires a higher temperature for the transition from the glassy state to the supercooled liquid state. In this case the expansion coefficient of free-volume in supercooled state $\alpha_{s c l}$ is significantly less than for higher filling (see Table I).

\section{Conclusion}

The study of confined 1-PrOH in the fully-filled mesopores of the SBA-15 matrix shows the shift of glass transition temperature $T_{g}$ as well as the onset temperature to plateau region $T_{b 2}$ to higher temperatures compared to bulk state.
The desorption of 1-PrOH from SBA-15 matrix at room temperature, at the decreased content of 1-PrOH in the matrix from $k=0.42$ down to 0.15 , generates gradually microcracks between molecules $1-\mathrm{PrOH}$. For $k=0.34$, this effect is not noticeable in the $v_{h}(T)$ dependence but for the lower filling of $k=0.25$, the effect is visible. From the $\tau_{4}(k)$ dependence we can conclude that 1-PrOH forms layers at the pore walls. Thickness of the layer decreases with lower of 1-PrOH content.

At low filling $k=0.15$, the temperature dependence of $v_{h}(T)$ exhibits the lowest expansion coefficient in supercooled state in comparison to the higher content of 1$\mathrm{PrOH}$. It is due to the strongly attached molecules to the polar surface, which are located in thin layer. Temperature dependences of $v_{h}(T)$ display the highest $T_{g}$ values compared to bulk state and confined systems with higher content of 1-PrOH.

The onset to the plateau at the temperature $T_{b 2}$ in the $v_{h}(T)$ dependences increases with decrease of the filling $k$ and the plateau effect disappeared at $k=0.15$.

\section{Acknowledgments}

Work was partially supported by projects VEGA 2/0030/16, VEGA 2/0157/17 and APVV-16-0369.

\section{References}

[1] J. Bartoš, H. Švajdlenková, O. Šauša, M. Lukešová, D. Ehlers, M. Michl, P. Lunkenheimer, A. Loidl, J. Phys. Condens. Matter 28, 015101 (2016).

[2] Dongyuan Zhao, Jianglin Feng, Qisheng Huo, N. Melosh, G.H. Fredrickson, B.F. Chmelka, G.D. Stucky, Science 279, 548 (1998).

[3] O. Šauša, I. Matko, E. Illeková, E. Macová, D. Berek, J. Phys. Conf. Series 618, 012041 (2015).

[4] I. Matko, O. Šauša, E. Macová, D. Berek, J. Therm. Anal. Calorim. 121, 163 (2015).

[5] E. Illeková, E. Macová, V. Majerník, I. Matko, O. Šauša, J. Therm. Anal. Calorim. 116, 753 (2014).

[6] O. Šauša, V. Majerník, E. Illeková, E. Macová, D. Berek, Acta Phys. Pol. A 125, 798 (2014).

[7] S. Takahara, Y. Yamamuro, H. Suga, J. NonCryst. Solids 171, 259 (1994).

[8] J. Kansy, Nucl. Instrum. Methods Phys. Res. A 374, 235 (1996).

[9] R. Zaleski, EELViS, accessed 15 June 2011. 